\title{
Ultrasound biomicroscopic measurement of development of anterior chamber angle
}

\author{
Hiroshi Kobayashi, Hisako Ono, Junichii Kiryu, Kaori Kobayashi, Takehisa Kondo
}

Department of Ophthalmology, Amagasaki Hospital, Amagasaki, Japan H Kobayashi H Ono

Department of Ophthalmology and Visual Science, Graduate School of Medicine Kyoto University, Kyoto, Japan

H Kobayashi

J Kiryu

Department of Ophthalmology, Osaka Red Cross Hospital, Osaka, Japan K Kobayashi

Department of Ophthalmology, Kobe City General Hospital, Kobe, Japan

T Kondo

Correspondence to: Hiroshi Kobayashi, Department of Ophthalmology, Amagasaki Hospital, 1-1-1 Higashi-daimotsu-cho, Amagasaki, Hyogo, 660, Japan.

Accepted for publication 25 November 1998

\begin{abstract}
Aim-To establish normative values for the anterior segment in normal infants and children in relation to age.

Methods-Anterior segments were measured in 46 normal infants and children ( 21 males and 25 females, aged from 1 to 60 months (mean 17.09 (SD 16.99) months)), by use of ultrasound biomicroscopy.

Results-Anterior chamber depth, trabecular-iris angle, angle opening (trabecular-iris) distances at 250 and $\mathbf{5 0 0}$ $\mu \mathrm{m}$ from the scleral spur, and the thickness of the thickest part of the iris were 1724-3473 $\mu \mathrm{m}$ (2505 (SD 480) $\mu \mathrm{m}), 15.35-$ $44.79^{\circ}\left(28.74(7.46)^{\circ}\right), 116-367 \mu \mathrm{m}(247.4$ (65.9) $\mu \mathrm{m}), 166-509 \mu \mathrm{m}(349.5(87.1) \mu \mathrm{m})$, and $249-579 \mu \mathrm{m}(434.6(74.6) \mu \mathrm{m})$, respectively. All factors in this study showed a significant correlation with logarithm of age $(r=0.937, \mathrm{p}=0.0001 ; r=0.867, \mathrm{p}=$ $0.0001 ; r=0.929, \mathrm{p}=0.0001 ; r=0.917, \mathrm{p}=$ $0.0001 ; r=0.748, p=0.0001$ ), and significantly correlated with each other.

Conclusions-Ultrasound biomicroscopy is a powerful tool for obtaining precise images and measurement of the development of the anterior segment in infants and children. Normative values were established for anterior segment dimensions in relation to age. Anterior chamber depth, trabecular-iris angle, angle opening distances at 250 and $500 \mu \mathrm{m}$ from the scleral spur, and iris thickness showed linear increases in relation to logarithm of age.

(Br f Ophthalmol 1999;83:559-562)
\end{abstract}

Precise in vivo measurements of the developing anterior segment, in particular of the angle, are essential to understand the mechanism of congenital and developmental glaucoma. ${ }^{12}$ Conventional methods of evaluating the anterior chamber angle, such as gonioscopy and ultrasonic biometry, may not accurately assess the dimensions of the anterior chamber, leading to incorrect diagnoses. ${ }^{3}$ Measurements should be used that are quantitative. The advantage of ultrasound biomicroscopic technology is its accuracy and reproducibility. ${ }^{4}$ High resolution ultrasound biomicroscopy provides precise measurements of the angle as well as information on the mechanism of various types of glaucoma..$^{5-13}$

We have developed a method for measuring the anterior segment of infants and obtaining live images by use of ultrasound biomicroscopy and a muscle hook with topical anaesthesia. ${ }^{14}$ Ultrasound biomicroscopy allows one to make precise measurements of the anterior segments of even premature neonates. It is encouraging that distinct images of the exact area of interest can be obtained by a simple control of the eyeball with a muscle hook. Even infants in poor medical condition can be examined in this way through the ports of the isolette. Our objective was to measure prospectively, for the first time, anterior chamber depth, angle width, and iris thickness in infants and children in order to develop normative values. Correlation of these variables with age, as well as with each other, was evaluated.

\section{Patients and methods}

PATIENTS

Forty six normal infants and children were selected for study. The study protocol and consent forms were approved by the human subjects committee on Amagasaki Hospital. Informed consent for each infant was obtained from at least one parent. Infants with malformations were excluded from the study, as were genetic disorders, suspected intrauterine infections, or retinopathy of prematurity greater than stage II.

ULTRASOUND BIOMICROSCOPIC MEASUREMENTS Ophthalmic and ultrasound biomicroscopic measurements were made of the right eye of each infant. Ultrasonic biomicroscope 840 (Zeiss-Humphrey, San Leandro, CA, USA) with a $50 \mathrm{~Hz}$ transducer and ultrasound biomicroscope UX-02 (Rion, Tokyo, Japan) with a $30 \mathrm{~Hz}$ transducer were used. Each infant or child was placed in a supine position. For infants, an infant Bangarter speculum, rather than an eye cup, was used to separate the eyelids after a drop of $0.5 \%$ proxymetacaine (proparacaine) had been applied. The palpebral fissure was filled with $2.5 \%$ methylcellulose as a coupling medium. Scanning was performed by manipulating the eyeball with a muscle hook without pressure on the eyeball and placing the probe close to the area of interest. We repeated each measurement three times to confirm reproducibility and eliminate artefacts.

Anterior chamber depth (ACD) was measured as an axial distance from the internal corneal surface to the lens surface. Ultrasound biomicroscopic cross sectional views through the angle region were obtained for most of the measurements, which were made in the temporal meridian; views were as vertical as possible, as determined from the screen image. The following variables were measured: ACD, 


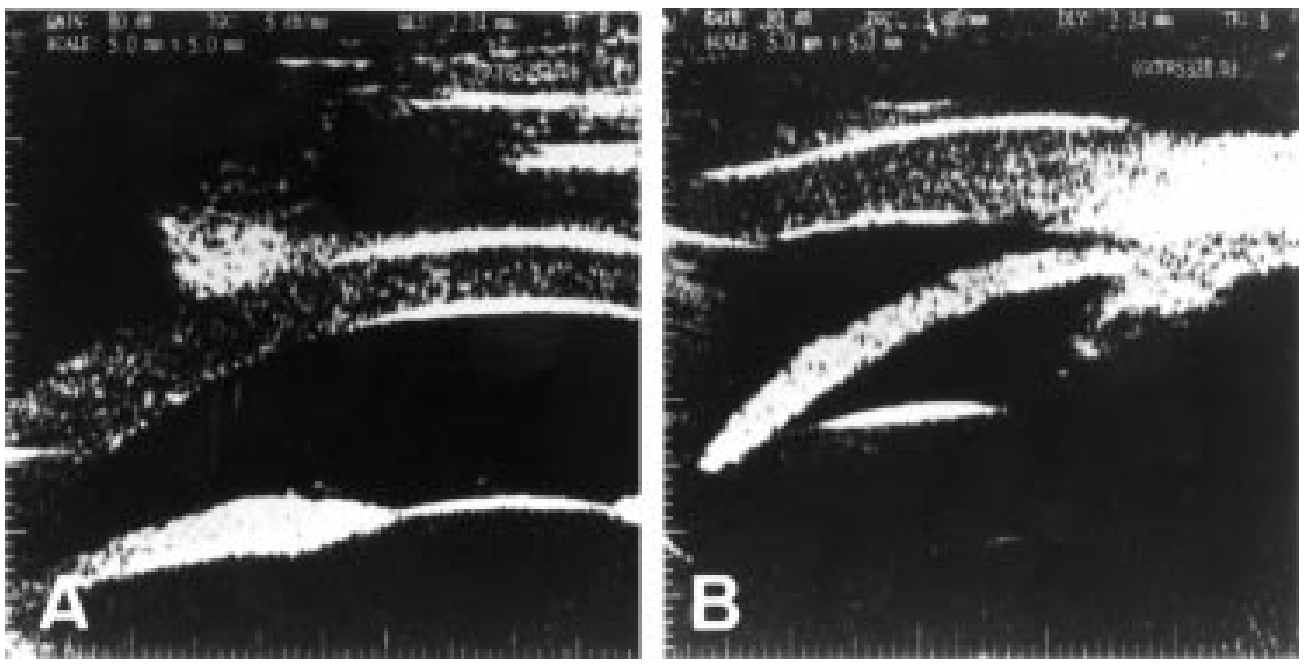

Figure 1 A 1 month old infant. (A) Ultrasound biomicroscopic image of the central anterior chamber. (B) Ultrasound biomicroscopic cross sectional view through the temporal angle region.
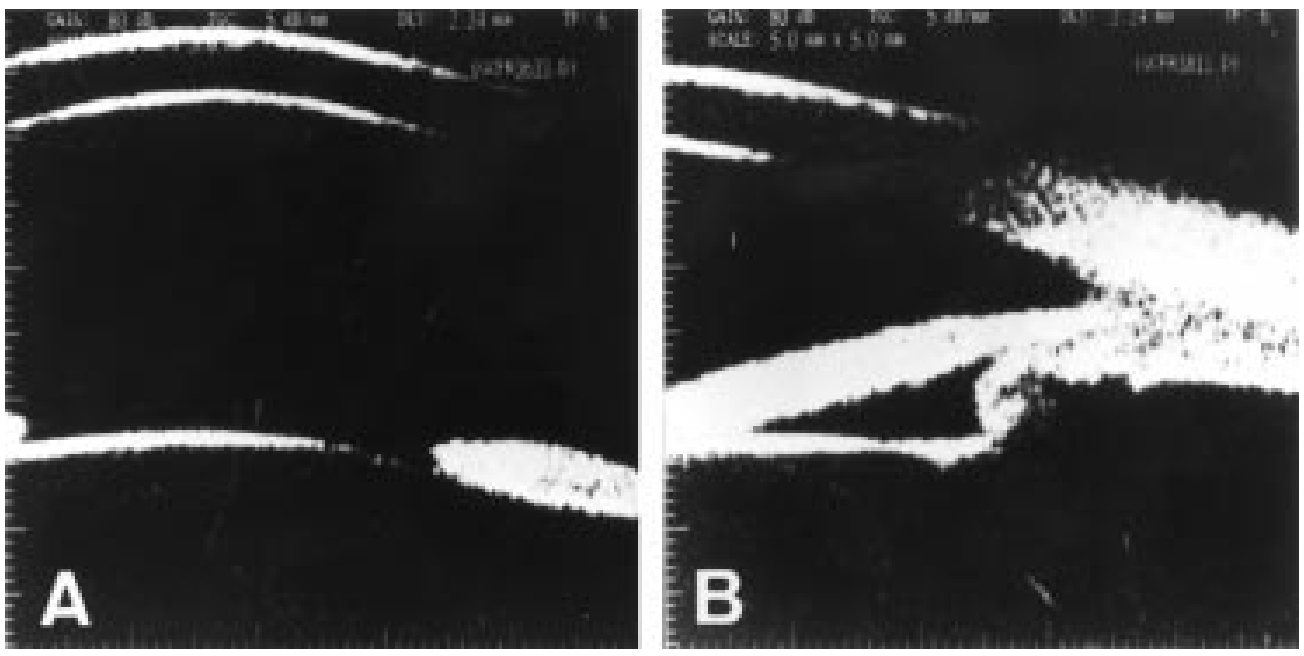

Figure 2 A 13 month old infant. (A) Ultrasound biomicroscopic image of the central anterior chamber. (B) Ultrasound biomicroscopic cross sectional view through the temporal angle region.

Table 1 Summary of the definition of variables in this study

\begin{tabular}{ll}
\hline Variables & Definition \\
\hline $\begin{array}{l}\text { Anterior chamber depth (ACD) } \\
\text { Trabecular-iris angle (TIA) }\end{array}$ & $\begin{array}{l}\text { Distance between the inner corneal surface and the lens surface } \\
\text { Angle between the arms passing through a point on the trabecular meshwork } 500 \mu \mathrm{m} \\
\text { from the scleral spur and the point perpendicularly opposite on the iris }\end{array}$ \\
Angle opening distance 250 (AOD250) & $\begin{array}{l}\text { Length of a perpendicular from the trabecular meshwork on the iris at a point } 250 \mu \mathrm{m} \\
\text { from the scleral spur }\end{array}$ \\
Angle opening distance 250 (AOD250) & $\begin{array}{l}\text { Length of a perpendicular from the trabecular meshwork on the iris at a point } 250 \mu \mathrm{m} \\
\text { from the scleral spur } \\
\text { Iris thickness at thickest part of the iris }\end{array}$ \\
\hline
\end{tabular}

Table 2 Mean, SD, and range of variables of anterior segment measurements in 46 infants and children

\begin{tabular}{lll}
\hline Variables & Mean $(S D)$ & Range \\
\hline Age $($ months $)$ & $17.09(16.99)$ & 1 to 60 \\
ACD $(\mu \mathrm{m})$ & $2505(480)$ & 1724 to 3473 \\
TIA $($ degree $)$ & $28.74(7.46)$ & 15.35 to 44.79 \\
AOD250 $(\mu \mathrm{m})$ & $247.4(65.9)$ & 116 to 367 \\
AOD500 $(\mu \mathrm{m})$ & $349.5(87.1)$ & 166 to 509 \\
Iris thickness $(\mu \mathrm{m})$ & $434.6(74.6)$ & 249 to 579 \\
\hline
\end{tabular}

$\mathrm{ACD}=$ anterior chamber depth; TIA=trabecular-iris angle; AOD250=angle opening distance at $250 \mu \mathrm{m}$ from scleral spur; AOD500 $=$ angle opening distance at $500 \mu \mathrm{m}$ from scleral spur; iris thickness=thickness at the thickest part of the iris. trabecular-iris angle (TIA), angle opening distance at 250 and $500 \mu \mathrm{m}$ from the scleral spur (AOD250 and AOD500), and iris thickness, as described by Palvin et al. ${ }^{67}$ Iris thickness was measured at the thickest part of the iris. A summary of the definition of factors is shown in Table 1.

STATISTICS

Values were expressed as mean (SD) and as a range. Regression analysis was performed to determine the precise relation of the ACD, TIA, AOD250, AOD500, and iris thickness to age. Because our interest is in the prediction of 

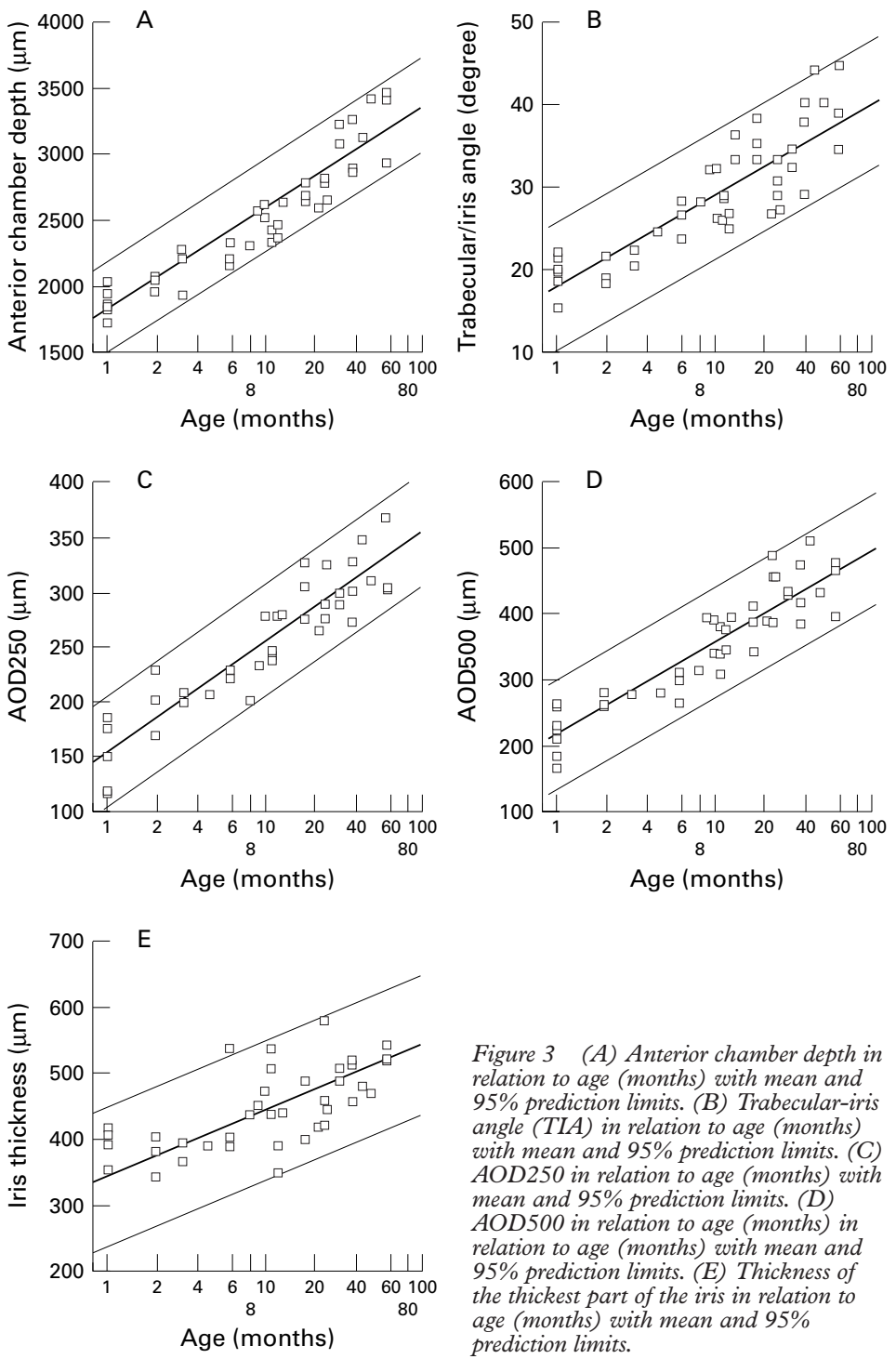

Figure 3 (A) Anterior chamber depth in relation to age (months) with mean and 95\% prediction limits. (B) Trabecular-iris angle (TIA) in relation to age (months) with mean and $95 \%$ prediction limits. (C) AOD250 in relation to age (months) with mean and $95 \%$ prediction limits. (D) AOD500 in relation to age (months) in relation to age (months) with mean and 95\% prediction limits. (E) Thickness of the thickest part of the iris in relation to age (months) with mean and 95\% prediction limits.

Table 3 Regression analysis and correlation coefficients of anterior chamber depth (ACD), trabecular-iris angle, AOD250, AOD500, and iris thickness in relation to age

\begin{tabular}{llll}
\hline Variable & $r$ & $p$ Value & Regression quotation \\
\hline ACD & 0.937 & 0.0001 & $1779.819+769.257 \times \log ($ age $($ month $))$ \\
TIA & 0.867 & 0.0001 & $18.162+11.206 \times \log ($ age $($ month) $)$ \\
AOD250 & 0.929 & 0.0001 & $151.539+101.528 \times \log ($ age $($ month $))$ \\
AOD500 & 0.917 & 0.0001 & $220.551+136.671 \times \log ($ age $($ month $))$ \\
Iris thickness & 0.748 & 0.0001 & $344.393+95.584 \times \log ($ age $($ month $))$
\end{tabular}

$\mathrm{ACD}=$ anterior chamber depth; TIA=trabecular-iris angle; AOD250=angle opening distance at $250 \mu \mathrm{m}$ from scleral spur; AOD $500=$ angle opening distance at $500 \mu \mathrm{m}$ from scleral spur; iris thickness=thickness at the thickest part of the iris.

Table 4 Regression analysis and correlation coefficients between anterior chamber depth (ACD), trabecular-iris angle, AOD250, AOD500, and iris thickness

\begin{tabular}{lllll}
\hline & $r$ & $p$ Value & Intercept & Slope \\
\hline ACD v TIA & 0.913 & 0.0001 & -6.918 & 0.140 \\
ACD v AOD250 & 0.877 & 0.0001 & -45.379 & 0.117 \\
ACD v AOD500 & 0.890 & 0.0001 & -55.646 & 0.162 \\
ACD v iris thickness & 0.667 & 0.0001 & 170.641 & 0.105 \\
TIA v AOD250 & 0.861 & 0.0001 & 36.072 & 7.352 \\
TIA v AOD500 & 0.856 & 0.0001 & 62.936 & 9.973 \\
TIA v iris thickness & 0.655 & 0.0001 & 246.667 & 6.540 \\
AOD250 v AOD500 & 0.923 & 0.0001 & 38.214 & 1.259 \\
AOD250 v iris thickness & 0.648 & 0.0001 & 247.320 & 0.757 \\
AOD500 v iris thickness & 0.673 & 0.0002 & 233.087 & 0.577 \\
\hline
\end{tabular}

$\mathrm{ACD}=$ anterior chamber depth; $\mathrm{TIA}=$ trabecular-iris angle; $\mathrm{AOD} 250=$ angle opening distance at $250 \mu \mathrm{m}$ from scleral spur; AOD500=angle opening distance at $500 \mu \mathrm{m}$ from scleral spur; iris thickness $=$ thickness at the thickest part of the iris. the relation for an individual infant, 95\% prediction limits were calculated for the eye measurements. These limits are wider than the $95 \%$ confidence limits for the mean eye measurement as given levels of predictor variables.

\section{Results}

Ultrasound biomicroscopic measurements of the anterior segment were obtained from 46 infants and children, 21 males and 25 females, aged from 1 to 60 months (mean 17.09 (SD 16.99) months).

Figure 1 shows the ultrasound biomicroscopic image of the central anterior chamber and cross sectional view through the temporal angle region of a 1 month old infant. The ACD, TIA, AOD250, AOD500, and thickness of the thickest part of the iris were $1551 \mu \mathrm{m}$, $15.35^{\circ}, 129 \mu \mathrm{m}, 220 \mu \mathrm{m}$, and $394 \mu \mathrm{m}$, respectively. Figure 2 shows ultrasound biomicroscopic image of the central anterior chamber and cross sectional view of a 13 month old infant. The ACD, TIA, AOD250, AOD500, and thickness of the thickest part of the iris were $2640 \mu \mathrm{m}, 46.38^{\circ}, 280 \mu \mathrm{m}, 394 \mu \mathrm{m}$, and $439 \mu \mathrm{m}$, respectively.

The ACD, TIA, AOD250, AOD500 and thickness of the thickest part of the iris for the group of infants and children are shown in Table 2. The ACD, TIA, AOD250, AOD500 and iris thickness were 1724-3473 $\mu \mathrm{m}(2505$ $(\mathrm{SD} 480) \mu \mathrm{m}), 15.35-44.79^{\circ}\left(28.74(7.46)^{\circ}\right)$, 116-367 $\mu \mathrm{m}$ (247.4 (65.9) $\mu \mathrm{m}), 166-509 \mu \mathrm{m}$ (349.5 (87.1) $\mu \mathrm{m})$, and 249-579 $\mu \mathrm{m}(434.6$ (74.6) $\mu \mathrm{m})$, respectively. The ACD, TIA, AOD250, AOD500, and iris thickness significantly correlated to age $(r=0.937, \mathrm{p}=0.0001$; $r=0.867, \mathrm{p}=0.0001 ; r=0.929, \mathrm{p}=0.0001 ; r$ $=0.917, \mathrm{p}=0.0001 ; r=0.748, \mathrm{p}=0.0001)$ (Fig 3 and Table 3). The slopes and intercepts for the regression analysis, with correlation coefficients with $\mathrm{p}$ values, are shown in Table 3.

The ACD was significantly correlated with TIA, AOD250, AOD500, and iris thickness $(r$ $=0.913, \mathrm{p}=0.0001 ; r=0.877, \mathrm{p}=0.0001 ; r=$ $0.890, \mathrm{p}=0.0001 ; \mathrm{r}=0.677, \mathrm{p}=0.0001)$, as shown in Table 4 . The TIA also showed a significant correlation with AOD250, AOD500, and iris thickness $(r=0.861, \mathrm{p}=0.0001 ; r=$ $0.856, \mathrm{p}=0.0001 ; r=0.655, \mathrm{p}=0.0001)$. The AOD250 was significantly correlated with AOD500 and iris thickness $(r=0.923, \mathrm{p}=$ $0.0001 ; r=0.648, \mathrm{p}=0.0001)$. There was a significant correlation between AOD500 and iris thickness $((r=0.673, \mathrm{p}=0.0002)$.

\section{Discussion}

We measured the anterior segments of infants and children, and established normative values and $95 \%$ prediction limits for anterior chamber depth, trabecular-iris angle, AOD250, AOD500, and iris thickness in relation to age. Anterior chamber depth, trabecular-iris angle, AOD250, and AOD500 showed a significant increase in relation to logarithm of age. Several investigators reported the development of the axial length and the anterior chamber depth. ${ }^{15-20}$ Sampolesi and Caruso ${ }^{1}$ and Kiskis et $a l^{21}$ demonstrated a significant correlation of 
axial length and corneal diameter with logarithm of age. The growth of the axial length and corneal diameter had been reported to be divided into three growth periods: a rapid natal phase in the first year and a half, the following slower infantile phase from the second to sixth year, and the final slow juvenile phase lasting until the age of 13 years. Our measurements also showed that the development of the anterior segment dimensions had a rapid growth phase from birth to approximately 1.5 years and a following slower growth phase.

General anaesthesia is usually required for a thorough examination of children under 5 years of age. ${ }^{21}$ The general condition of some infants often precludes examination under general anaesthesia. The opacity of anterior media and iridocorneal abnormalities, which are frequently seen in patients with developmental glaucoma, prevent observation with conventional methods such as slit lamp biomicroscopy. To overcome the difficulties, we have developed a method for measuring the anterior segment of neonates and obtaining live images by use of ultrasound biomicroscopy and a muscle hook with topical anaesthesia. ${ }^{14}$ Ultrasound biomicroscopy allows one to make precise measurements of the anterior segments even in premature neonates.

It is well known that the diagnosis of congenital glaucoma and the consequent indication of surgical intervention depend on clinical symptoms and intraocular pressure. Sampolesi and Caruso and Reibakdi showed that, in cases with borderline pressure levels that do not show any meaningful deviation from the normal, axial length measurements with ultrasonography are helpful in treating those patients. ${ }^{119}$ Kiskis et al reported that the corneal diameter was a more reliable guide than the axial length in the assessment of congenital glaucoma. ${ }^{20}$ In eyes with congenital glaucoma, the corneal diameter increases more than the axial length. In our preliminary study, we measured anterior segments of patients with congenital glaucoma and found that their ACD, TIA, AOD250, and AOD500 were greater than upper $95 \%$ prediction limits. The use of the ultrasound biomicroscopy would facilitate the evaluation of anterior segments. However, the measurements of the dimensions of the anterior segments is needed in cases with borderline pressure levels.

We established normative values for anterior segment in infants and children. These data provide the first information on the develop- ment of the anterior segments. The normative values should greatly aid ophthalmologists in evaluating anterior segment dimensions of infants and children who are suspected of having congenital glaucoma and congenital anomalies.

1 Sampaolesi R, Caruso R. Ocular echometry in the diagnosis of congenital glaucoma. Arch Ophthalmol 1982;100:574-7.

2 Tarkkanen A, Uusitalo R, Mianowicz J. Ultrasonographic biometry in congenital glaucoma. Acta Ophthalmol 1983; 61:618-23.

3 Makabe R. Vergleichende Untersuchungen der Kammerwinkelweite mit Echographie und Gonioskopie. Klin Monatsbl Augenheilkd 1989;194:6-9.

4 Tello C, Liebmann J, Potash SD, et al. Measurement of ultrasound biomicroscopy images: intraobserver and interobserver reliability. Invest Ophthalmol Vis Sci 1994;35: 3549-52.

5 Pavlin CJ, Sherar MD, Foster FS. Subsurface ultrasound microscopic imaging of the intact eye. Ophthalmology 1990; 97:244-50.

6 Pavlin CJ, Harasiewicz K, Sherar MD, et al. Clinical use of ultrasound biomicroscopy. Ophthalmology 1991;98:28795.

7 Pavlin CJ, Harasiewicz K, Foster FS. Ultrasound biomicroscopy of anterior segment structures in normal and glaucomatous eyes. Am f Ophthalmol 1992;113:381-9.

8 Pavlin CJ, McWhae JA, McGowan HD, et al. Ultrasound biomicroscopy of anterior segment tumors. Ophthalmology 1992;99:1220-8.

9 Pavlin CJ, Ritch R, Foster FS. Ultrasound biomicroscopy in plateau iris syndrome. Am f Ophthalmol 1992;113:390-5.

10 Tello C, Chi T, Shepps G, et al. Ultrasound biomicroscopy in pseudophakic malignant glaucoma. Ophthalmology 1993; 100:1330-4

11 Potash SD, Tello C, Liebmann J, et al. Ultrasound biomicroscopy in the pigment dispersion syndrome. Ophthalmology 1994;101:332-9.

12 Aslanides IM, Libre PE, Silverman RH, et al. High frequency ultrasound imaging in pupillary block glaucoma. Br f Ophthalmol 1995;79:972-6.

13 Kobayashi H, Kobayashi K, Kiryu J, et al. Ultrasound biomicroscopic analysis of the effect of pilocarpine on the anterior chamber angle. Graefes Arch Clin Exp Ophthalmol 1997;235:425-30.

14 Kobayashi H, Kobayashi K, Kiryu J, et al. Ultrasound biomicroscopic measurements of anterior chamber angle in premature infants. Br f Ophthalmol 1997;81:460-4.

15 Larsen JS. The sagittal growth of the eye. I. Ultrasonic measurement of the depth of the anterior chamber from measurement of the depth of the anterior chamber
birth to puberty. Acta Ophthalmol 1971;49:239-62.

16 Larsen JS. The sagittal growth of the eye. II. Ultrasonic measurement of the axial diameter of the lens and the anterior segment from birth to puberty. Acta Ophthalmol 1971; 49:427-40.

17 Larsen JS. The sagittal growth of the eye. IV. Ultrasonic measurement of the axial length of the eye from birth to puberty. Acta Ophthalmol 1971;49:873-86.

18 Grignolo A, Rivara A. Biometry of the human eye from the sixth month of pregnancy to tenth year of life (measurements of the axial length, retinoscopy refraction, total refraction, corneal and lens refraction). In: Vanysek J, ed. Diagnostica ultrasonica in ophthalmologica. II Symposium Diagnostica ultrasonica in ophthalmologica. II Symposium 1968. Universita JE Purkyne, Brno, Czechoslovakia (Opus1968. Universita JE Purkyne, Brno, Czechoslovakia (Opuscula Ophthath

19 Reibakdi A. Biometric ultrasound in the diagnosis and follow-up of congenital glaucoma. Ann Ophthalmol 1982; 14:807-8

20 Kiskis AA, Markowitz SN, Morin JD. Corneal diameter and axial length in congenital glaucoma. Can f Ophthamol 1985;20:93-7.

21 Dickens DJ, Hoskins HDJr. Diagnosis and treatment of congenital glaucoma. In: Ritch R, Shields MB, Krupin T, eds. The glaucomas. 2nd ed. St Louis: Mosby, 739-49. 